\title{
OPEN Microbiota and transcriptome changes of Culex pipiens pallens larvae exposed to Bacillus thuringiensis israelensis
}

\author{
Ruiling Zhang ${ }^{1,2 \bowtie}$, Wenjuan Liu ${ }^{1,2}$, Qian Zhang ${ }^{1,2}$, Xinyu Zhang ${ }^{1,2}$ \& Zhong Zhang ${ }^{1,2 \bowtie}$
}

Culex pipiens pallens is an important vector of lymphatic filariasis and epidemic encephalitis. Mosquito control is the main strategy used for the prevention of mosquito-borne diseases. Bacillus thuringiensis israelensis (Bti) is an entomopathogenic bacterium widely used in mosquito control. In this study, we profiled the microbiota and transcriptional response of the larvae of $C x$. pipiens pallens exposed to different concentrations of $B t i$. The results demonstrated that $B t i$ induced a significant effect on both the microbiota and gene expression of $C x$. pipiens pallens. Compared to the control group, the predominant bacteria changed from Actinobacteria to Firmicutes, and with increase in the concentration of $B t i$, the abundance of Actinobacteria was gradually reduced. Similar changes were also detected at the genus level, where Bacillus replaced Microbacterium, becoming the predominant genus in Bti-exposed groups. Furthermore, alpha diversity analysis indicated that Bti exposure changed the diversity of the microbota, possibly because the dysbiosis caused by the Bti infection inhibits some bacteria and provides opportunities to other opportunistic taxa. Pathway analysis revealed significant enhancement for processes associated with sphingolipid metabolism, glutathione metabolism and glycerophospholipid metabolism between all Bti-exposed groups and control group. Additionally, genes associated with the Toll and Imd signaling pathway were found to be notably upregulated. Bti infection significantly changed the bacterial community of larvae of $C_{x}$. pipiens pallens.

Mosquitoes cause enormous public health risks by transmitting various infectious agents of diseases ${ }^{1}$. More than one-half of the population of the world is threatened by mosquito-borne diseases ${ }^{2-4}$, causing a huge burden to human health and the economy ${ }^{5}$. Culex pipiens pallens is one of the sibling species of the Culex pipiens complex and the most prevalent Culex species in northern China. As the primary vector of lymphatic filariasis, epidemic encephalitis and other diseases, this mosquito poses a serious threat to human health ${ }^{6-8}$. Due to the lack of effective vaccines and increasing drug resistance in pathogens, vector control is an important tool for the prevention of major mosquito-borne diseases worldwide ${ }^{9,10}$. As a rapid, convenient and effective method of controlling mosquitoes, chemical insecticide is a core component for mosquito-borne disease control programs and has significantly reduced the prevalence of vector-borne diseases around the world ${ }^{11}$. However, the long-term largescale use of chemical insecticides can result in many deleterious effects, including selection for insect resistance to pesticides, morbidity of non-target insects, and devastating effects on the environment and ecosystems ${ }^{12-15}$, which have become a major obstacle to the control of mosquito-borne diseases.

Bacillus thuringiensis $(B t)$ is an entomopathogenic bacterium widely used in biocontrol of insect pests. Extensive studies have revealed that the toxicity of $B t$ is induced by parasporal crystalline proteins protoxins synthesized during sporulation. When activated by proteolytic cleavage in the midgut after ingestion by target larvae, the active toxins bind to midgut receptors and ultimately form pores that destabilize osmotic balance if cells of the midgut lining, leading to death of the host ${ }^{16,17}$. The high level of specificity of $B t$ subspecies toward insects has also been proposed to be associated with microbiota of its hosts ${ }^{18,19}$. Some studies revealed that host-associated microbiota might be required for effective $B t$ killing of its insect hos $\mathrm{t}^{20}$. Others found that a bacteriocin produced by $B t$ can inhibit the growth of some bacteria ${ }^{21}$.

${ }^{1}$ Collaborative Innovation Center for the Origin and Control of Emerging Infectious Diseases, Shandong First Medical University (Shandong Academy of Medical Sciences), Taian, China. ${ }^{2}$ School of Basic Medical Science, Shandong First Medical University, Taian, China. ${ }^{\circledR}$ email: zhangrl_06@126.com; vector0518@126.com 
Hundreds of $B t$ subspecies have been described to date, and each of these subspecies produces a specific set of toxins as a crystal during sporulation, and the crystals have been successfully used for the control of agricultural pests and medical vectors ${ }^{21,22}$. Among all of the $B t$ subspecies, parasporal crystalline proteins generated by the bacterium $B$. thuringiensis israelensis (Bti) are toxic when ingested by mosquitoes. The World Health Organization (WHO) has proposed the use of Bti for the prevention and control of arboviral diseases ${ }^{23}$. As a powerful and environmentally friendly biological insecticide ${ }^{24,25}, B t i$ has been widely used since 1976 and exhibits a specific biolarvicidal effect for controlling species of the Culicidae family ${ }^{26-28}$. Although the action of Bti has been extensively studied, the underlying mechanisms and relationships with microbiota of hosts are far from being resolved. The microbiota of the mosquito is highly variable, is shaped by many factors such as environment and life history traits ${ }^{29-32}$ and can actively participate in defense against pathogens ${ }^{33-35}$. It is important to discriminate changes in the microbial community, which might be helpful in revealing the association of microbiota with Bti toxicity. In addition, differentially expressed genes and molecular pathways induced by Bti infection might contribute to better understanding of the molecular regulation of the mosquito response to Bti and help in evaluating potential targets for molecular-based vector control. In the present study, which is based on the $16 \mathrm{~S}$ rRNA gene and RNA sequencing (RNA-Seq), we explored the dynamic changes in the microbiota of $C x$. pipiens pallens that are exposed to different Bti concentrations and identified the differentially expressed genes triggered by the Bti infection.

\section{Material and methods}

Mosquito rearing. Culex pipiens pallens colony used in this study were collected from Shandong Province and maintained in the laboratory for years. Mosquitoes were maintained in a rearing room at $27 \pm 1{ }^{\circ} \mathrm{C}$ and $65 \%$ relative humidity $(\mathrm{RH})$ with a daily photoperiod of 14:10 h (L:D). The larvae were reared in dechlorinated tap water in plastic containers and fed with a slurry of pork liver powder (homemade) and yeast mixed with distilled water. Pupae were collected and placed into a beaker and then reared in insect cages $(35 \times 35 \times 35 \mathrm{~cm})$ until adults emerged. Newly emerged adults were provided with $10 \%$ sugar solution ad libitum. Adult females of 4-5 days old were supplied a diet of mouse blood. Mouse was fixed on a plastic board and put into the insect cages. Experimental protocol for all animal experiments was in accordance with guidelines and with permission from the local animal ethics committee and with permission from the Institutional Animal Care and Use Committee of Shandong First Medical University. The study was carried out in compliance with the ARRIVE guidelines $^{36}$. Fully engorged females laid eggs in oviposition cups, and the eggs were transferred into plastic containers with dechlorinated tap water. All samples were flash frozen in liquid nitrogen immediately following collection and then stored at $-80^{\circ} \mathrm{C}$ until for subsequent uses.

Bti larvicide. The larvicide used in this study was liquid suspension of Bacillus thuringiensis israelensis (Lukang, Shandong, China). The concentrations used were referred to manufacturer's introduction (2.5 ml larvicide $/ 97.5 \mathrm{ml}$ water). Application concentrations were set to Group A (0.5 ml larvicide $/ 99.5 \mathrm{ml}$ water), Group B (1 ml larvicide/99 ml water), Group C (1.5 ml larvicide/98.5 ml water) and Group D (100 ml water) as control group. Two hundred eggs of Cx. pipiens pallens were put into each treatment (Group A, B, C and D), respectively.

The larvicidal activity of different Bti concentrations was performed on third instar larvae. Twenty larvae were placed in $100 \mathrm{ml}$ beaker that containing appropriate concentration of $B t i$ solutions that corresponding to Group A, B, C and D, respectively. Mortality was assessed after $24 \mathrm{~h}$, during which larvae were fed to avoid the influences caused by starvation. The assays were done in five replicates. The percentage of larval mortality was calculated for each concentration using Abbott's formula ${ }^{37}$. In addition, dilutions of the stock were cultured on Potato Dextrose Agar (PDA) medium to evaluate spore counts of each Bti concentration.

DNA extraction and 16S rRNA library preparation. Fifteen fourth instar larvae were randomly collected from each group (with three biological replicates for each group). All samples were surface sterilized with $75 \%$ ethanol for $5 \mathrm{~s}$ and then washed with sterile water for three times. Genomic DNA was extracted from the whole body of mosquitoes using DNeasy Blood and Tissue Kit (QIAGEN, United States) following the manufacturer's instructions. DNA concentration and purity were monitored on $1 \%$ agarose gels. Each DNA sample was diluted to $1 \mathrm{ng} / \mu \mathrm{l}$ using sterile water. The V4 hypervariable region of the $16 \mathrm{~S}$ rRNA gene was amplified using the primers 515F (5'-TCGTCGGCAGCGTCAGATGTGTATAAGAGACAGGTGCCAGCMGCCGCGGTAA-3') and 806R (5'-GTCTCGTGGGCTCGGAGATGTGTATAAGAGACAGGGACTACHVGGGTWTCTAAT-3'). All PCR reactions were carried out with $15 \mu \mathrm{l}$ of PCR Master Mix (New England Biolabs), including $0.2 \mu \mathrm{M}$ of primers and $10 \mathrm{ng}$ DNA template. Thermal cycling consisted of the initial denaturation for $1 \mathrm{~min}\left(98^{\circ} \mathrm{C}\right)$, followed by 30 cycles of denaturation at $98^{\circ} \mathrm{C}$ for $10 \mathrm{~s}$, annealing at $50{ }^{\circ} \mathrm{C}$ for $30 \mathrm{~s}$, and elongation at $72{ }^{\circ} \mathrm{C}$ for $30 \mathrm{~s}$ with final extension for $5 \mathrm{~min}\left(72^{\circ} \mathrm{C}\right)$. PCR products were detected in $2 \%$ agarose gel and then purified with the Qiagen Gel Extraction Kit (Qiagen, Germany). Sequencing libraries were generated using a DNA PCRFree sample preparation kit (Illumina, USA) following the manufacturer's recommendations. The quality of the library was assessed on the Qubit 2.0 Fluorometer (Thermo Scientific) and Agilent Bioanalyzer 2100 system. The resulting libraries were sequenced on an Illumina NovaSeq6000 platform.

16S rRNA gene sequence data analysis. Paired-end 16S reads were merged using FLASH V1.2. $7^{38}$. QIIME 2 (release 2018.8) ${ }^{39}$ was used to filter the raw reads and obtain high-quality clean reads. Chimera sequences were identified and removed by compared clean tags with the Silva database (https://www.arbsilva.de $/)^{40}$ using the UCHIME algorithm ${ }^{41}$. Uparse v7.0.1001 ${ }^{42}$ was used to perform sequences analysis, and sequences with $\geq 97 \%$ similarity were assigned to the same OTUs. The taxonomy of a representative sequence of 
each OTU was annotated by comparison to the Silva Database based on the mothur algorithm. Taxonomy summaries of relative abundance data at different taxonomic levels were generated.

Alpha (within-sample) and beta (between-sample) diversity was calculated with QIIME 2 and displayed with $\mathrm{R}$ software V 2.15.3. Chao1 (richness estimators) measures diversity based on only the number of OTUs present, whereas Shannon (diversity indices) considers both abundance and evenness of the OTUs in a community. Good's Coverage was used to provide a description of rarefaction in terms of the total sampled OTU diversity ${ }^{43}$. Overlapping and core microbiota of each sample were shown in Venn diagrams, which were generated by use of a web tool (http://bioinformatics.psb.ugent.be/webtools/Venn/). Principal coordinate analysis (PCoA) was performed to obtain principal coordinates and visualize complex multidimensional data.

RNA extraction, library construction and sequencing. Total RNA was extracted from 15 fourth instar larvae that were randomly selected from each Bti concentration group and control group (with three biological replicates for each group) using the RNAiso plus reagent (TaKaRa, Japan). RNA degradation and contamination were checked with denaturing agarose gel electrophoresis, and a NanoDrop (ND-1000) spectrophotometer (NanoDrop, USA) was used to test the quality and quantity of the purified RNA. The integrity of RNA was assessed by $1.5 \%$ agarose gel electrophoresis.

Transcriptome sequencing libraries were generated using an RNA Library Prep Kit for Illumina (NEB, USA) according to the manufacturer's instructions. mRNA was purified from total RNA using poly-T oligo-attached magnetic beads and was fragmented into short fragments using divalent cations under elevated temperature in NEBNext First Strand Synthesis Reaction Buffer (5X). First strand cDNA was synthesized using random hexamer primers and mRNA templates. Second strand cDNA synthesis was subsequently synthesized using DNA polymerase I and RNase H, dNTPs and buffer. After end reparation and adenylation of the 3' ends, short fragments were connected using the NEBNext Adaptor with a hairpin loop structure. The library fragments were purified with an AMPure XP system (Beckman Coulter, Beverly, USA) for selection of suitable fragments after PCR amplification. Finally, the PCR products were purified (AMPure XP system) and the library quality was assessed on a Bioanalyzer 2100 (Agilent, USA). High-throughput transcriptome sequencing was performed on an Illumina NovaSeq platform.

RNA-Seq data analysis. Firstly, the quality and number of the reads of each sample were assessed using FastQC v 0.11.44. Fastq files were trimmed using Trimmomatic v $0.38^{45}$. Clean data (clean reads) were obtained by removing adapter, poly- $\mathrm{N}$ and low-quality reads from the raw data. Then, the Q30 and GC content was calculated based on the clean data. The paired-end clean reads were aligned to the reference genome using Hisat 2 $\mathrm{v} 2.1 .0^{46}$. The genome sequences of $C x$. pipiens pallens (PRJNA702155) were chosen as reference genes. The reads of each sample mapped to reference genes were assembled by StringTie v1.3.3b ${ }^{47}$. Then, the fragments Per Kilobase of the transcript sequence per millions mapped reads (FPKM) base pairs of each gene were calculated based on the length of the gene and read counts mapped to this gene.

Differential expression analysis of samples was performed using the DESeq2 R package (1.16.1). $P$ values of the results were adjusted using the Benjamini and Hochberg's approach for controlling the false discovery rate. Genes with an adjusted $P$ value $<0.05$ were assigned as differentially expressed genes (DEGs). Kyoto Encyclopedia of Genes and Genomes (KEGG) pathway and Gene Ontology (GO) enrichment analyses of differentially expressed genes was implemented by the clusterProfiler R package, in which gene length bias was corrected. GO terms and KEGG pathways with corrected $P$ value less than 0.05 were considered significantly enriched in differential expressed genes.

\section{Results}

Microbiota diversity. A total of 1,064,820 16S rRNA gene sequences were generated, 80,377 to 99,826 reads from different samples (SRA: PRJNA675318). After quality control, 778,775 reads were used for the following analysis and clustered into 886 OTUs, including 844 bacteria, 32 archaea and 10 unclassified. Together, the bacterial OTUs were associated with 20 unique phyla, 34 classes, 79 orders, 144 families, 252 genera and 158 species. There were 208 shared OTUs among the four experimental groups, and 80, 87, 11 and 129 unique OTUs were detected in each group, respectively (Fig. 1, Table S1). Firmicutes was the dominant phylum of mosquito samples exposed to Bti, while the predominant phylum in the control groups was Actinobacteria (Fig. 2). The abundance of Actinobacteria decreased gradually with increasing Bti concentrations (Fig. 2). Similarly, this tendency was also detected at the genus level. Microbacterium was the predominant genus in the control group. However, the abundance of Microbacterium was significantly reduced in all Bti-exposed groups, and the abundance was lower with an increase in Bti concentration. In addition, Bacillus became the dominant genus in all Bti-exposed groups (Fig. S1).

Good's coverage ranged from 99.9 to $100 \%$ for each sample, suggesting that the identified $16 \mathrm{~S}$ rDNA sequences were sufficient to fully represent the bacterial diversity of the samples in this study (Table 1). Consistent with the decreased trend in $\mathrm{LC}_{50}$ and $\mathrm{LC}_{90}$ values in Group A, B, and C (Table S2), the Chaol index decreased with increasing Bti concentrations, indicating that high concentrations of Bti reduced the bacterial richness of $C x$. pipiens pallens. Furthermore, the Chaol index revealed that the bacterial richness of the control group was higher than group C. Both group A and B had higher microbial richness than the control group (Fig. 3A). Comparison of the Shannon index between groups exposed to Bti with the control group demonstrated a significant increase in the diversity in the Bti-exposed groups (Fig. 3B). The structure of bacterial communities between groups was evaluated with PCoA analysis. The weighted UniFrac PCoA showed that $63.83 \%$ (axis 1) and 23.52\% (axis 2) of the bacterial composition variation among the four groups were explained (Fig. 4). All samples exposed to Bti clustered separately from the control group, indicating that the microbiota of $C x$. pipiens pallens was significantly 


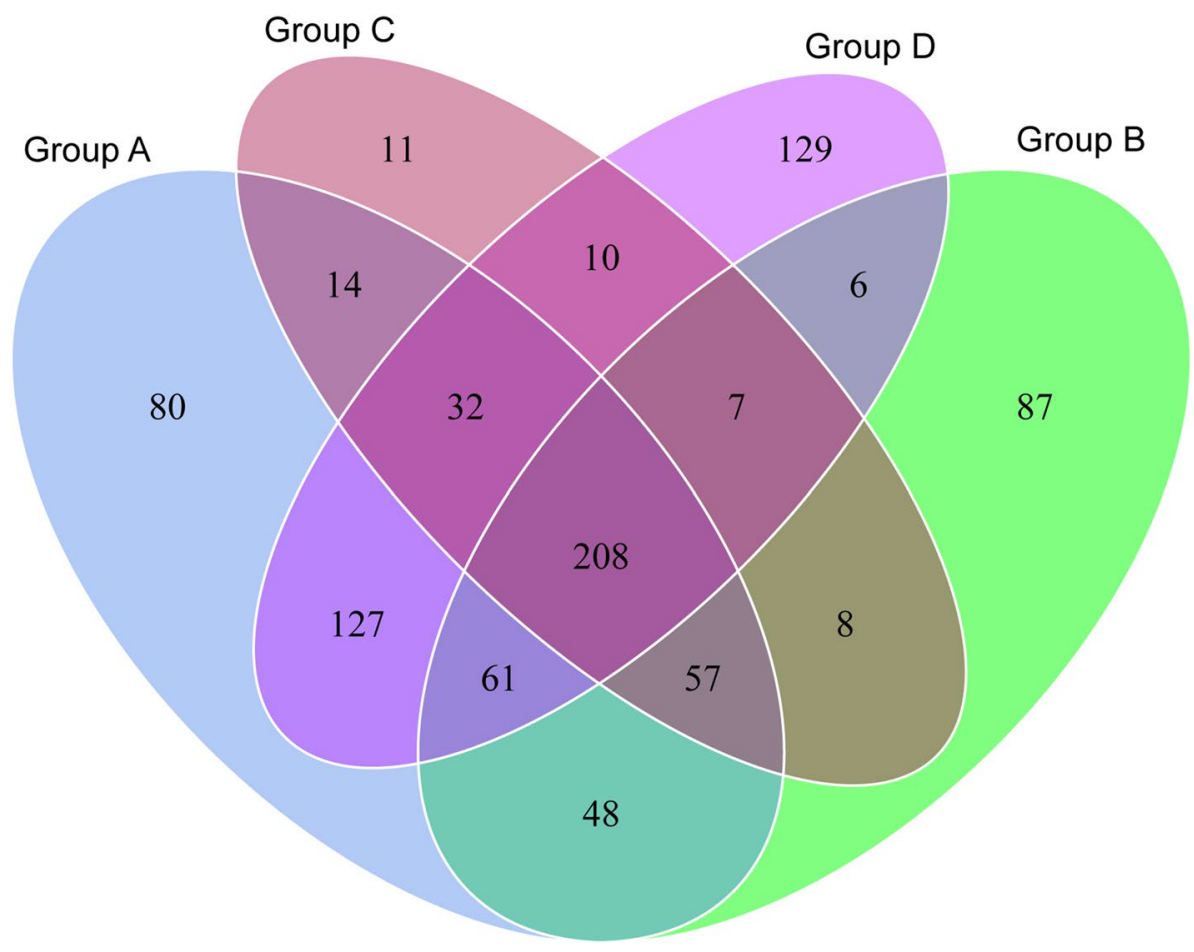

Figure 1. Venn diagram showing the number of OTUs shared between four groups.

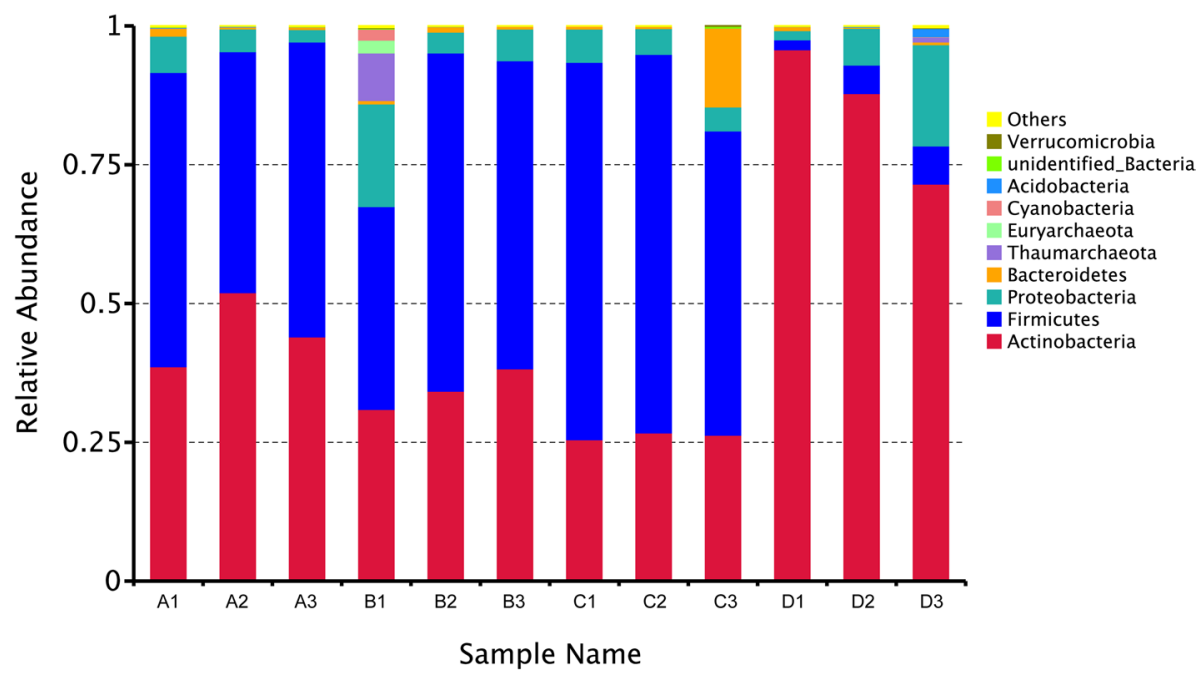

Figure 2. Bar plot of the relative abundances of microbial community at the rank of phyla level.

changed upon exposure to Bti. Furthermore, the samples exposed to different Bti concentrations clustered separately, conforming that the different bacterial communities exhibit a pattern of specialization (Fig. 4).

RNA-seq. All RNA-seq data have been deposited at SRA (NCBI) with the accession number PRJNA675746. Altogether, 18,078 genes were identified, and 15,959 genes were annotated according to the referenced genome. To test the effect of $B t i$ on $C x$. pipiens pallens gene regulations, DEG analyses were carried out for groups exposed to $B t i$ compared to their expression in the control group, and between groups exposed to different Bti concentrations. To gain an initial overview of the data, heatmaps of all the samples were generated (Fig. 5). A major variation in color pattern was visually identifiable between the control group and the groups exposed to Bti (Fig. 5). A total of 1,208 genes were identified as differentially expressed between Group A and D, among which 566 genes were upregulated, and 1,336 genes were downregulated (Table 2; Fig. 6). When the gene expression levels were compared between Group B and D, 2,277 genes were differentially expressed, 1,251 genes and 1,026 genes were upregulated and downregulated, respectively (Table 2; Fig. 6). A total of 1,538 differentially expressed genes were 


\begin{tabular}{|c|c|c|c|c|c|}
\hline Bti concentration & Sample name & Observed species & Shannon & \begin{tabular}{|l|} 
Chaol \\
\end{tabular} & Goods coverage \\
\hline \multirow{3}{*}{3 ITU } & $\mathrm{A} 1$ & 484 & 2.497 & 518.348 & 0.999 \\
\hline & $\mathrm{A} 2$ & 321 & 1.91 & 385.208 & 0.999 \\
\hline & A3 & 250 & 1.962 & 298.158 & 0.999 \\
\hline \multirow{3}{*}{9 ITU } & B1 & 338 & 3.45 & 358.128 & 0.999 \\
\hline & B2 & 330 & 2.26 & 371.902 & 0.999 \\
\hline & B3 & 155 & 1.715 & 178.786 & 0.999 \\
\hline \multirow{3}{*}{$15 \mathrm{ITU}$} & $\mathrm{C} 1$ & 205 & 1.859 & 229.241 & 0.999 \\
\hline & $\mathrm{C} 2$ & 252 & 1.651 & 279.051 & 0.999 \\
\hline & $\mathrm{C} 3$ & 203 & 3.291 & \begin{tabular}{|l|}
227.7 \\
\end{tabular} & 0.999 \\
\hline \multirow{3}{*}{0 ITU } & D1 & 175 & 0.698 & 184.255 & 0.999 \\
\hline & $\mathrm{D} 2$ & 274 & 1.194 & 311.652 & 0.999 \\
\hline & D3 & 459 & 2.643 & 517.184 & 0.999 \\
\hline
\end{tabular}

Table 1. Details of alpha diversity indexes of four groups.
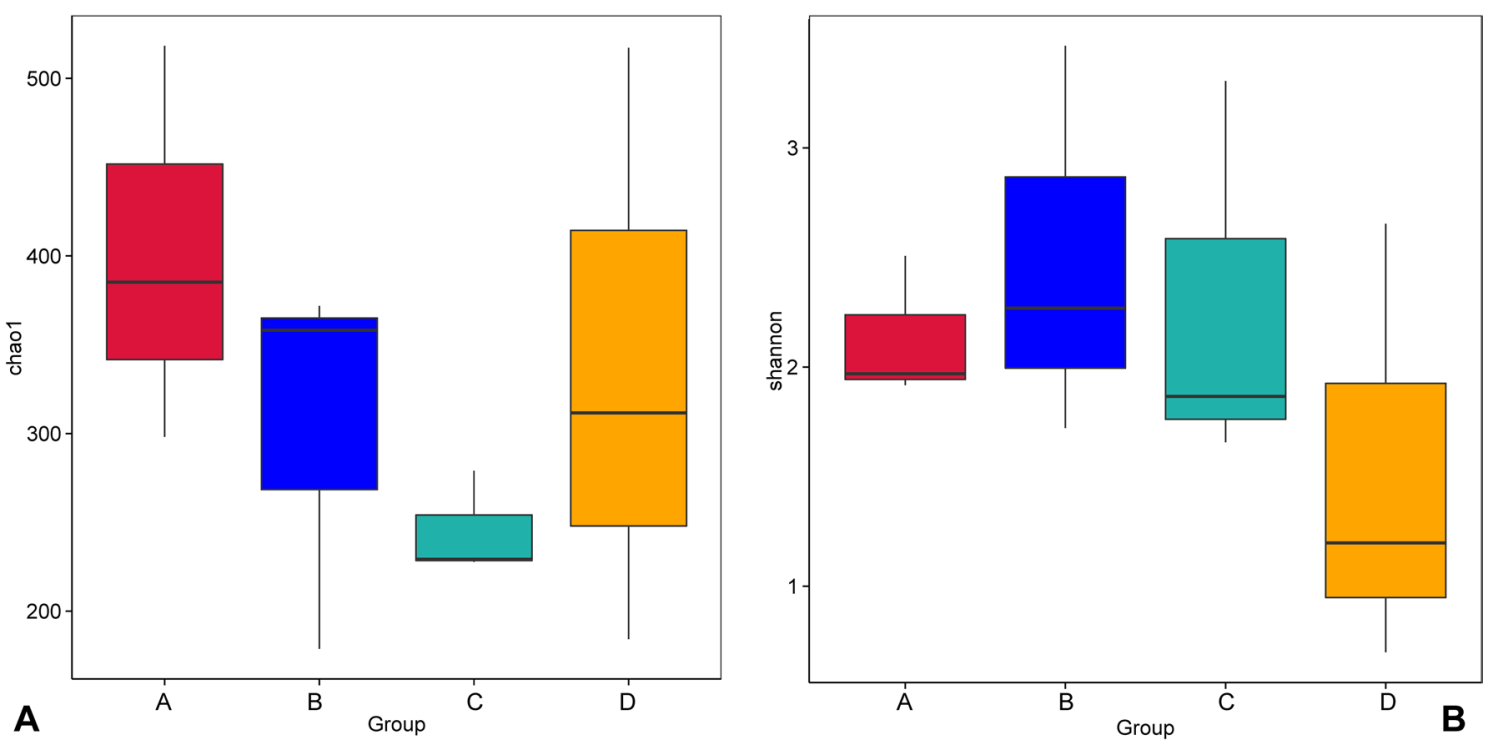

Figure 3. Boxplot representation of chaol (A) and Shannon (B) indexes.

found between Group C and D, including 783 upregulated genes and 755 downregulated genes (Table 2; Fig. 6). In contrast, there were substantially fewer differentially expressed genes in Groups A, B and C, suggesting fewer changes at the molecular level in these groups (Table 2).

The potential function of each DEGs was explored using GO and KEGG analyses. In the GO classification, the DEGs in all comparisons ( $\mathrm{A}$ vs B, A vs C, A vs D, B vs C, B vs D, C vs D) were divided into three main categories, including biological processes, cellular components, and molecular functions. The enriched functional terms of the DEGs demonstrated that molecular function was the most varied cluster between the groups exposed to $B t i$ and the control group (Fig. S2). In the KEGG analysis, the top 20 pathways were included in the subsequent analysis, and the metabolism pathways were the most enriched, followed by genetic information processing, environmental information processing, cellular processes, organismal systems, and human diseases (Table S3). 'Sphingolipid metabolism', 'glutathione metabolism' and 'glycerophospholipid metabolism' were three metabolism pathways presented in all comparison groups (A vs D, B vs D, C vs D); and 'DNA replication' of genetic information processing, 'ABC transporters' of environmental information processing, 'Toll and imd signaling pathway' of organismal systems were also found in three comparison groups. Furthermore, 'drug metabolism' of the metabolism pathway was detected only in B vs D and C vs D comparisons; 'autophagy' of cellular processes pathway was involved in the A vs D and B vs D groups, while 'apoptosis' was found in the C vs D groups (Fig. 7). Additionally, the KEGG pathway enrichment analysis demonstrated that the 'lysosome', 'autophagy' and 'glutathione metabolism' pathway were significantly enriched for the downregulated genes corresponding to the A vs D, B vs D, C vs D groups, respectively. Furthermore, the 'DNA replication' pathway was significantly enriched in upregulated genes in all three comparison groups (Fig. 7).

Compared with the control group, the genes in the drug metabolism (cytochrome P450) pathway were significantly downregulated in all three groups exposed to Bti (Table S4). A large number of Toll and imd signaling pathway genes were upregulated in the three comparison groups (A vs D, B vs D, C vs D). Only three 


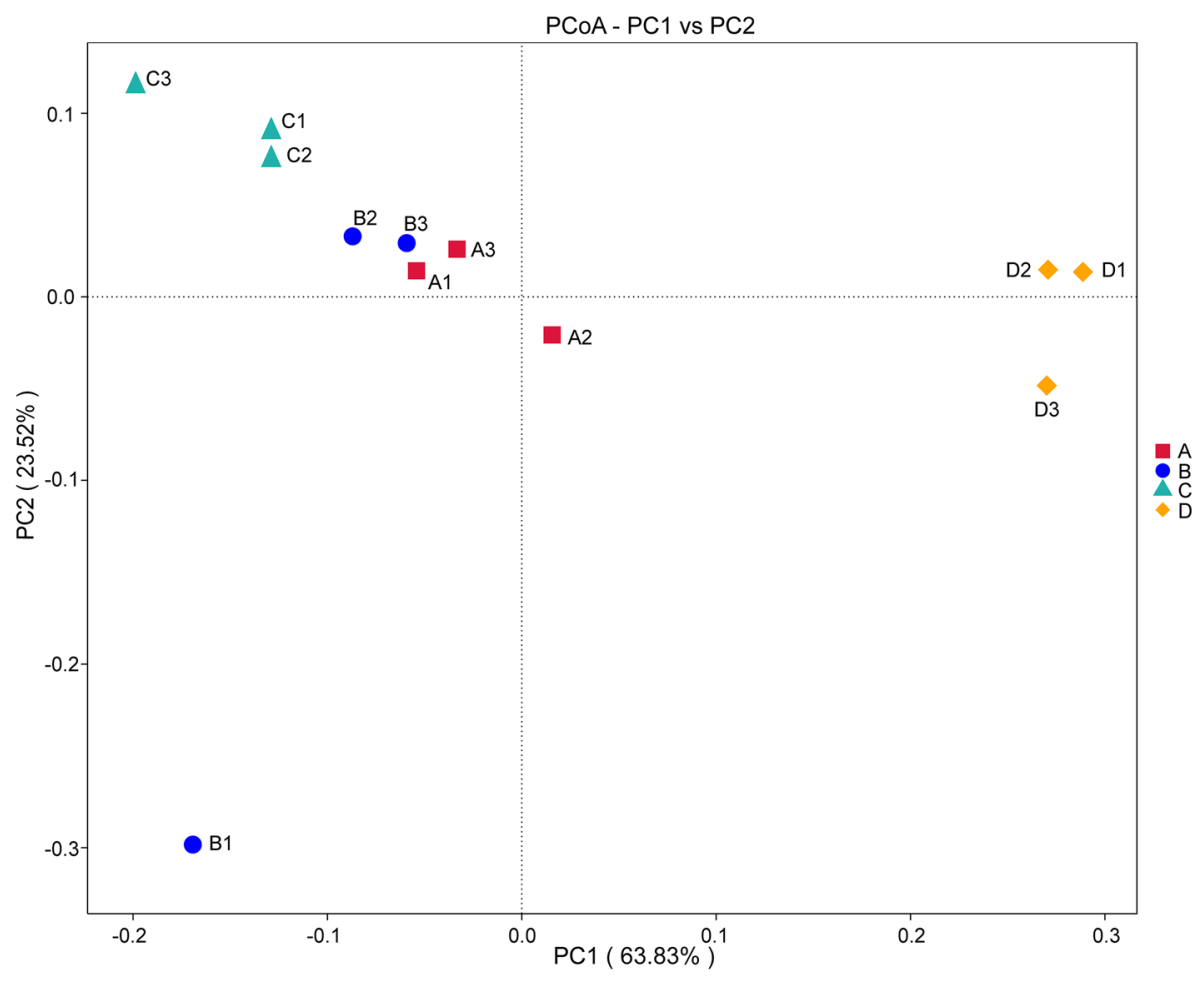

Figure 4. Principal coordinate analysis $(\mathrm{PCoA})$ of the microbial communities from different groups.

genes $(6044929,6052047$, and novel.497) were found to be downregulated. The autophagy pathway involved 10 upregulated genes and 14 downregulated genes (Table S4).

\section{Discussion}

Mosquito-borne diseases constitute a major burden for public health problems worldwide ${ }^{10,48,49}$.

The application of Bti to kill mosquito larvae is an effective strategy for mosquito control and safety for the environment $^{33,50,51}$. However, the underlying mechanism of Bti to kill mosquitoes has thus far not been completely elucidated.

Following the infection process of $B t i$, both the insect immune system and microbiota were changed. The activated immune response and dysbiosis of the microbiota have an important effect on the pathogenicity of $B t i^{52}$. A better understanding of the abundance and compositional changes of bacteria could potentially be helpful to illuminating the interaction between Bti toxicity and microbiota, exploring available bacterial taxa strengthening $B t i$ toxicity to mosquitoes, which finally contributes to the development of new sustainable mosquito control strategies. In the current study, the predominant bacteria of all CX. pipiens pallens larvae exposed to Bti was changed from Actinobacteria to Firmicutes, and the abundance of Actinobacteria was decreased with increase in the Bti concentrations (Fig. 2). Results of alpha diversity analysis suggested that Chaol indexes of Group A and B were higher than Chaol indexes of Group D, while Group C was lower than Group D; Shannon indexes of all groups exposed to Bti were exceeded Shannon indexes of Group D. Previous studies have proved that $B t$ can inhibit the growth of bacteria by producing bacteriocins ${ }^{28}$, which are extracellular peptides or proteins with bactericidal and bacteriolytic effects. For instance, cell wall hydrolyzing enzymes produced by bacteriocins, nisin and Pep5, can cause rapid lysis of staphylococcal ${ }^{53,54}$. In addition, some bacteria can also degrade toxins of $B t^{33,55,56}$. Based on the present results, we can deduce that Bti might cause imbalance of the microbiota of $C x$. pipiens pallens and reduced abundance of Actinobacteria, while some opportunistic bacteria, like Firmicutes, grow rapidly to render the increase of bacterial diversity and changed abundance of bacteria of larvae infected with Bti. However, due to the competition between Bti and microbiota, high toxicity of Bti finally causes reduction of bacteria richness.

In addition to the competitive relationships between $B t i$ and microbiota, beneficial interactions were also reported. Insects exhibited increased tolerance to Bti toxicity after removal of gut bacteria with antibiotics ${ }^{20,57,58}$. Bacteria of Helicoverpa armigera, a lepidopteran pest, have been proven produce proteases that contribute to the activation of $B t i$ protoxins into toxins $\mathrm{s}^{59}$. Reasons for the rapid increase of Firmicutes in samples exposed to $B t i$ are worthy of further investigation to determine whether these bacteria play roles similar to their roles in $H$. armigera or whether they can degrade Bti toxins and guarantee that mosquitoes survive despite dysbiosis. Results of PCoA analysis demonstrated that microbiota samples exposed to Bti clustered separately from the control group, and the samples exposed to different concentrations of Bti clustered separately (Fig. 4). Therefore, we can conclude that Bti exposure changes the bacterial composition of $C x$. pipiens pallens and the microbiota characteristics are determined by the Bti concentration which they were infected. 


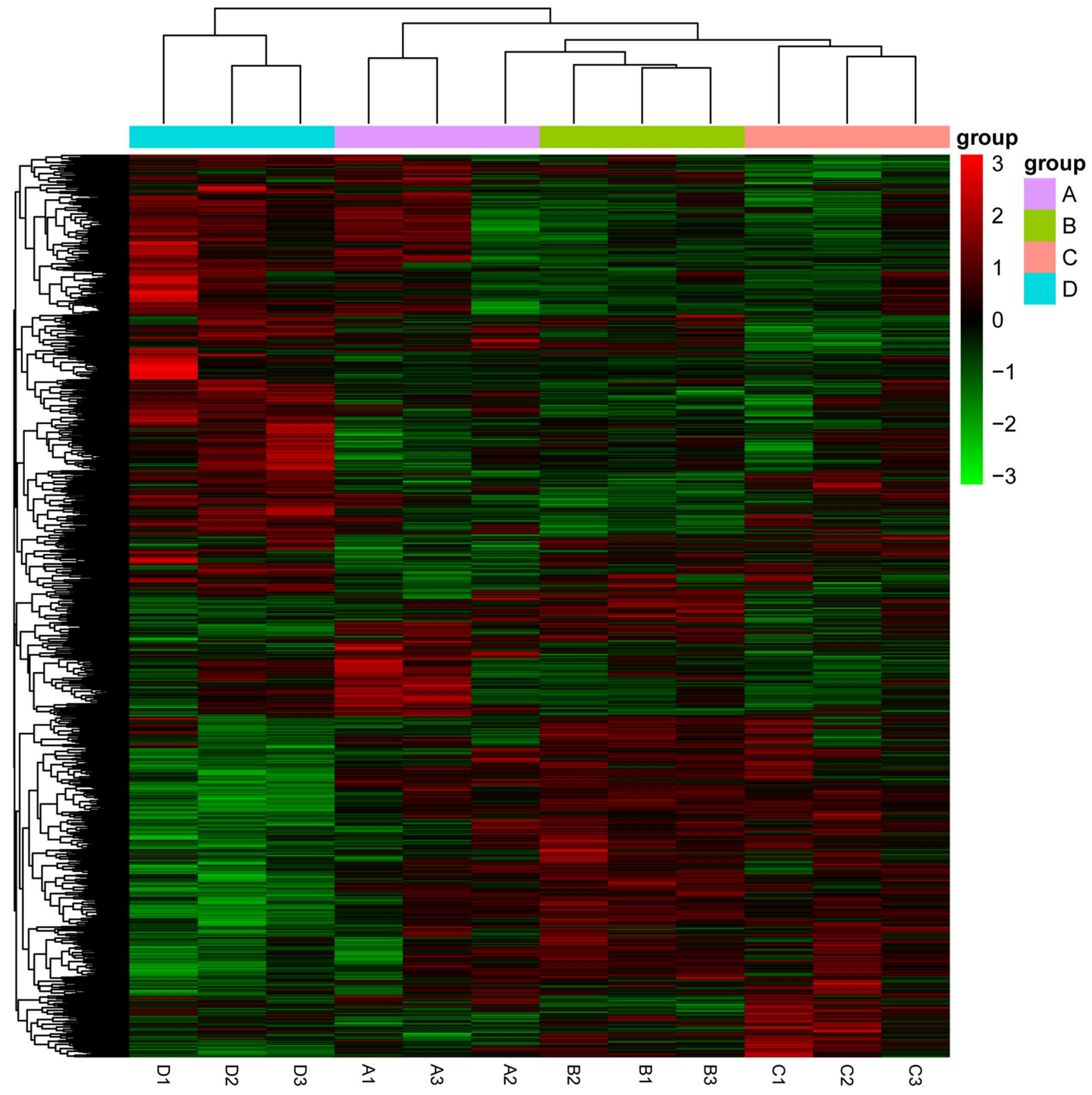

Figure 5. Heatmap showing the expression levels of differentially expressed genes (DEGs) in four groups.

\begin{tabular}{|l|l|c|c|}
\hline Groups & All & Up & Down \\
\hline A vs D & 1208 & 566 & 642 \\
\hline B vs D & 2277 & 1251 & 1026 \\
\hline C vs D & 1538 & 783 & 755 \\
\hline A vs B & 784 & 324 & 460 \\
\hline A vs C & 726 & 441 & 285 \\
\hline B vs C & 542 & 318 & 224 \\
\hline
\end{tabular}

Table 2. Number of differentially expressed genes between different groups. The threshold value of significance was DESeq2 p value $\leq 0.05 \mid \log 2$ FoldChange $\mid \geq 0.0$. 


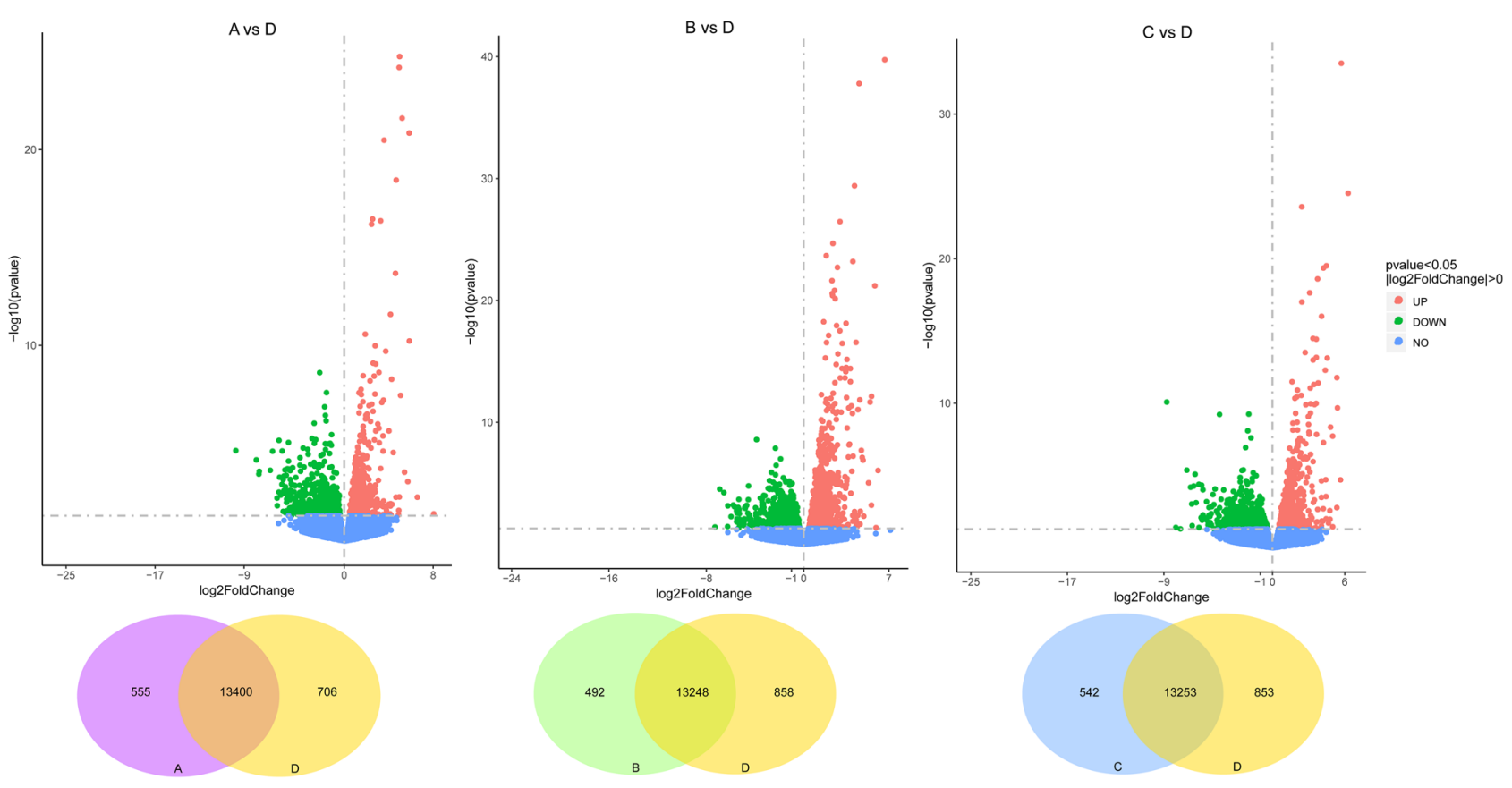

Figure 6. Scatter diagrams exhibiting differentially expressed gene profiling comparisons between group D and other three Bti exposed groups. A, Group A vs Group D; B, Group B vs Group D; C, Group C vs Group D. Blue point, non-significant difference in gene; red point: up-regulated gene; green point: down-regulated gene.

explored. Comparisons between Bti-exposed groups and control groups revealed that Bti induced a significant effect on the gene expression of $C x$. pipiens pallens larvae. Overall, there was no obvious difference in gene expression among groups exposed to different concentrations of $B t i$, while more than 1200 DEGs were identified as differentially expressed when comparing each of the Bti-exposed groups with the control group (Table 2). The pathway analysis revealed a significant enrichment of genes in processes associated with sphingolipid metabolism, glutathione metabolism and glycerophospholipid metabolism, which were found in all the comparison groups (A vs D, B vs D, C vs D). These three pathways are usually involved in forming of membranes and helping host defense against pathogens ${ }^{60,61}$. The overrepresentation of these pathways might reflect the damage caused by $B t i$ and the defense reaction that was activated against this. Consistent with these results, we also found enriched genes associated with the Toll and imd signaling pathway, which are two major immune pathways critical for the immune response that functions through the production of antimicrobial peptides (AMPs). AMPs are among the important members involved in humoral immune responses of insects ${ }^{52}$. The pronounced upregulation of gene expression associated with the Toll and imd signaling pathway indicated that larvae exposed to Bti mount a strong defense.

Though the killing mechanism of Bti has yet to be fully elucidated, its infections can cause tissue lesions and dysregulation of the gut environments for insects, and the microbiota residents in the host midgut trigger lethal septicemia ${ }^{62,63}$. Accordingly, we can hypothesize that the pathological damage and dysbiosis caused by Bti infection might activate protection mechanisms and lead to systematic variations in the expression of different genes. Therefore, the enrichment of drug metabolism (B vs D, C vs D groups), autophagy (A vs D, B vs D groups) and apoptosis ( $\mathrm{C}$ vs $\mathrm{D}$ group) pathways in different comparison groups might reflect the differing extent of the damages and subsequent self-protection activity of the larvae, which are determined by the different levels of Bti toxicity to which they were exposed.

\section{Conclusions}

Overall, in this study differences both in the microbiota and gene expression between samples exposed to different concentrations of $B t i$ and control groups were observed. These data may constitute a starting point, which further suggests that bacteria play important roles in the immune reaction of mosquitoes. More studies are needed to reveal potential associations between the relative abundances of certain taxa in the microbiota, host gene expression levels and corresponding pathways. The identification of specific candidate bacteria and genes related to defense reactions might enhance our understanding of the interactions between Bti and mosquitoes, which will provide novel insights into the development of mosquito control strategies. 

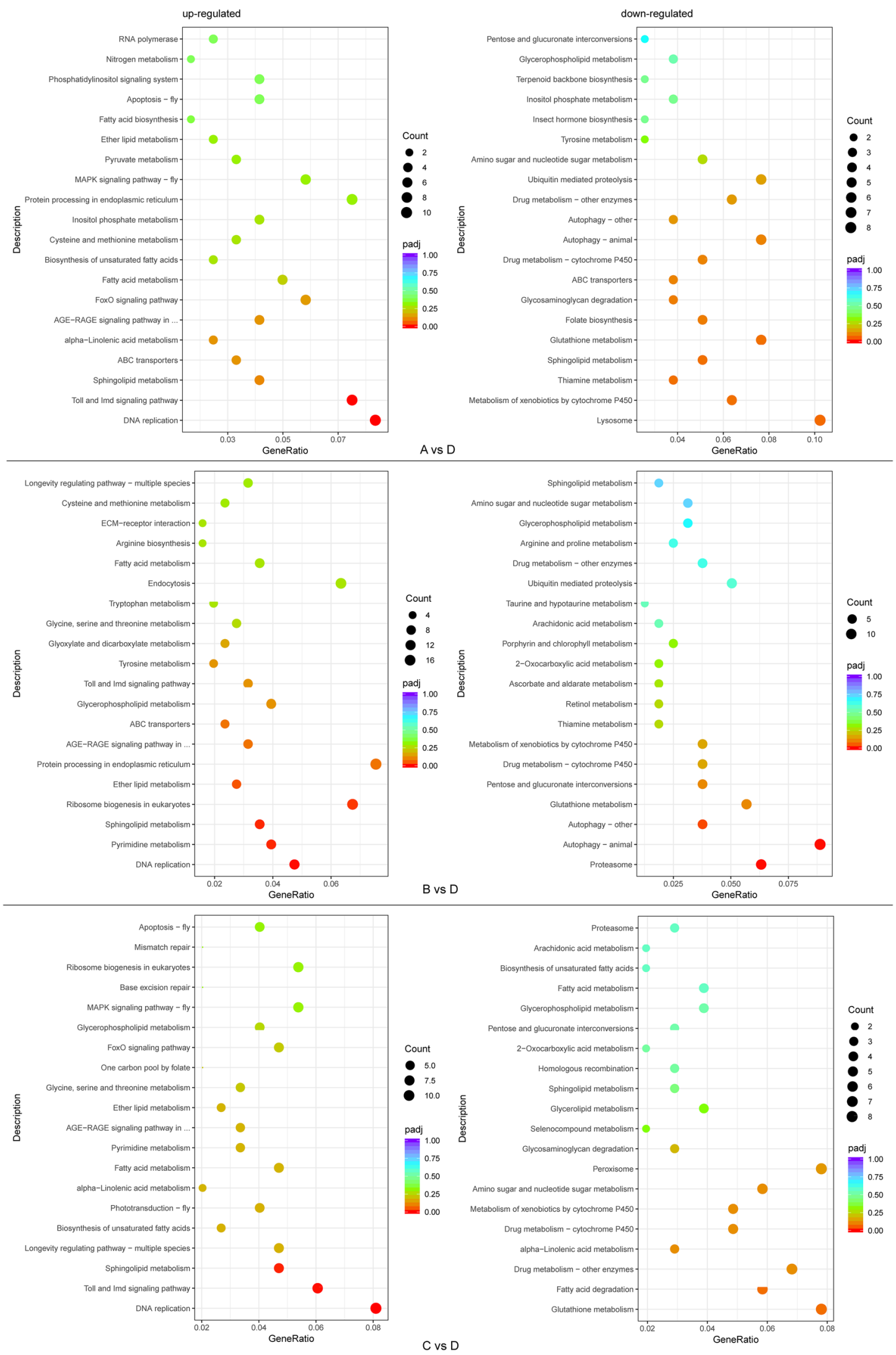

Figure 7. KEGG pathway enrichment analysis of up- and down-regulated DEGs. 
Received: 19 July 2021; Accepted: 29 September 2021

Published online: 12 October 2021

\section{References}

1. Acevedo, M. A. et al. Spatial heterogeneity, host movement and mosquito-borne disease transmission. PLoS One 10(6), e0127552 (2015).

2. Gething, P. W. et al. A new world malaria map: Plasmodium falciparum endemicity in 2010. Malar. J. 10, 378 (2011).

3. Gething, P. W. et al. A long neglected world malaria map: Plasmodium vivax endemicity in 2010. PLoS Negl. Trop. Dis. 6(9), e1814 (2012).

4. Bhatt, S. et al. The global distribution and burden of dengue. Nature 496(7446), 504-507 (2013).

5. Varmus, H. et al. Grand challenges in global health. Science 302, 398-399 (2003).

6. Fonseca, D. M., Smith, J. L., Kim, H. C. \& Mogi, M. Population genetics of the mosquito Culex pipiens pallens reveals sex-linked asymmetric introgression by Culex quinquefasciatus. Infect. Genet. Evol. 9, 1197-1203 (2009).

7. Turell, M. J. Members of the Culex pipiens complex as vectors of viruses. J. Am. Mos. Control Assoc. 28, 123-126 (2012).

8. Cano, J. et al. The global distribution and transmission limits of lymphatic filariasis: Past and present. Parasit. Vectors 7, 466 (2014).

9. van den Berg, H. et al. Global trends in the use of insecticides to control vector-borne diseases. Environ. Health. Perspect. 120(4), 577-582 (2012).

10. Chareonviriyaphap, T. et al. Review of insecticide resistance and behavioral avoidance of vectors of human diseases in Thailand. Parasit. Vectors 6, 280 (2013).

11. Ranson, H. \& Lissenden, N. Insecticide resistance in African Anopheles mosquitoes: A worsening situation that needs urgent action to maintain malaria control. Trends Parasitol. 32, 187-196 (2016).

12. Cui, F., Raymond, M. \& Qiao, C. L. Insecticide resistance in vector mosquitoes in China. Pest Manage. Sci. 62, 1013-1022 (2006).

13. Rivero, A., Vézilier, J., Weill, M., Read, A. F. \& Gandon, S. Insecticide control of vector-borne diseases: When is insecticide resistance a problem?. PLoS Pathog. 8, e1001000 (2010).

14. van den Berg, H. et al. Status of pesticide management in the practice of vector control: A global survey in countries at risk of malaria or other major vector-borne diseases. Malar. J. 10, 125 (2011).

15. Wang, Z. M. et al. Detection and widespread distribution of sodium channel alleles characteristic of insecticide resistance in Culex pipiens complex mosquitoes in China. Med. Vet. Entomol. 26, 228-232 (2012).

16. Lacey, L. Bacillus thuringiensis serovariety israelensis and Bacillus sphaericus for mosquito control. J. Am. Mosq. Control Assoc. 23, 133-163 (2007).

17. Vachon, V., Laprade, R. \& Schwartz, J. L. Current models of the mode of action of Bacillus thuringiensis insecticidal crystal proteins: A critical review. J. Invertebr. Pathol. 111(1), 1-12 (2012).

18. de Maagd, R. A., Bravo, A. \& Crickmore, N. How Bacillus thuringiensis has evolved specific toxins to colonize the insect world. Trends Genet. 17(4), 193-199 (2001).

19. de Maagd, R. A., Bravo, A., Berry, C., Crickmore, N. \& Schnepf, H. E. Structure, diversity, and evolution of protein toxins from spore-forming entomopathogenic bacteria. Annu. Rev. Genet. 37, 409-433 (2003).

20. Broderick, N. A., Raffa, K. F. \& Handelsman, J. Midgut bacteria required for Bacillus thuringiensis insecticidal activity. Proc. Natl. Acad. Sci. USA 103(41), 15196-15199 (2006).

21. Joung, K. B. \& Côté, J. C. A single phylogenetic analysis of Bacillus thuringiensis strains and bacilli species inferred from 16S rRNA gene restriction fragment length polymorphism is congruent with two independent phylogenetic analyses. J. Appl. Microbiol. 93, 1075-1082 (2002).

22. Berry, C. \& Crickmore, N. Structural classification of insecticidal proteins-towards an in silico characterisation of novel toxins. J. Invertebr. Pathol. 142, 16-22 (2016).

23. Gómez-Vargas, W., Valencia-Jiménez, K., Correa-Londoño, G. \& Jaramillo-Yepes, F. Novel larvicide tablets of Bacillus thuringiensis var. israelensis: Assessment of larvicidal effect on Aedes aegypti (Diptera: Culicidae) in Colombia. Biomedica 38, 95-105 (2018).

24. Rydzanicz, K., Sobczyński, M. \& Guz-Regner, K. Comparison of the activity and persistence of microbial insecticides based on Bacillus thuringiensis israelensis and Bacillus sphaericus in organic polluted mosquito breeding sites. Pol. J. Environ. Stud. 19, 1317-1323 (2010).

25. Zequi, J. A. C., Dos Santos, F. P. \& Lopes, J. Control of Culex quinquefasciatus and Cx. saltanensis (Diptera: Culicidae) with Bacillus thuringiensis israelensis in wastewater treatment lagoons. Rev. Colomb. Entomol. 40, 98-103 (2014).

26. Becker, N., Zgomba, M., Ludwig, M., Petric, D. \& Rettich, F. Factors influencing the activity of Bacillus thuringiensis var. israelensis treatments. J. Am. Mosq. Control Assoc. 8, 285-289 (1992).

27. Fillinger, U., Knols, B. G. J. \& Becker, N. Efficacy and efficiency of new Bacillus thuringiensis var. israelensis and Bacillus sphaericus formulations against Afrotropical anophelines in Western Kenya. Trop. Med. Int. Health. 8, 37-47 (2003).

28. Cherif, A., Rezgui, W., Raddadi, N., Daffonchio, D. \& Boudabous, A. Characterization and partial purification of entomocin 110 , a newly identified bacteriocin from Bacillus thuringiensis subsp. Entomocidus HD110. Microbiol. Res. 163(6), 684-692 (2008).

29. Wang, Y., Gilbreath, T. M., Kukutla, P., Yan, G. \& Xu, J. Dynamic gut microbiome across life history of the malaria mosquito Anopheles gambiae in Kenya. PLoS One 6, e0024767 (2011).

30. Gimonneau, G. et al. Composition of Anopheles coluzzii and Anopheles gambiae microbiota from larval to adult stages. Infect. Genet. Evol. 28, 715-724 (2014).

31. Muturi, E. J. et al. Mosquito microbiota cluster by host sampling location. Parasit. Vectors 11, 468 (2018).

32. Bascuñán, P., Niño-Garcia, J. P., Galeano-Castañeda, Y., Serre, D. \& Correa, M. M. Factors shaping the gut bacterial community assembly in two main Colombian malaria vectors. Microbiome 6, 1-12 (2018).

33. Patil, C. D., Borase, H. P., Salunke, B. K. \& Patil, S. V. Alteration in Bacillus thuringiensis toxicity by curing gut flora: Novel approach for mosquito resistance management. Parasitol. Res. 112(9), 3283-3288 (2013).

34. Soltani, A., Vatandoost, H., Oshaghi, M. A., Enayati, A. A. \& Chavshin, A. R. The role of midgut symbiotic bacteria in resistance of Anopheles stephensi (Diptera: Culicidae) to organophosphate insecticides. Pathog. Glob. Health 111(6), 289-296 (2017).

35. Dada, N., Sheth, M., Liebman, K., Pinto, J. \& Lenhart, A. Whole metagenome sequencing reveals links between mosquito microbiota and insecticide resistance in malaria vectors. Sci. Rep. 8(1), e1006539 (2018).

36. Percie du Sert, N. et al. The ARRIVE guidelines 2.0: Updated guidelines for reporting animal research. J. Cereb. Blood Flow Metab. 40, 1769-1777 (2020).

37. Abbott, W. S. A method of computing the effectiveness of an insecticide. J. Ecol. Entomol. 18, 265-266 (1925).

38. Magoč, T. \& Salzberg, S. L. FLASH: Fast length adjustment of short reads to improve genome assemblies. Bioinformatics 27, 2957-2963 (2011).

39. Caporaso, J. G. et al. QIIME allows analysis of high-throughput community sequencing data. Nat. Methods 7(5), 335-336 (2010).

40. Quast, C. et al. The SILVA ribosomal RNA gene database project: Improved data processing and web-based tools. Nucleic Acids Res. 41(Database issue), D590-596 (2013).

41. Edgar, R. C., Haas, B. J., Clemente, J. C., Quince, C. \& Knight, R. UCHIME improves sensitivity and speed of chimera detection. Bioinformatics 27(16), 2194-2200 (2011).

42. Edgar, R. C. UPARSE: Highly accurate OTU sequences from microbial amplicon reads. Nat. methods 10, 996-998 (2013). 
43. Good, I. J. The population frequencies of species and the estimation of population parameters. Biometrika 40, 237-264 (1953).

44. Andrews, S. FastQC: A quality control tool for high throughput sequence data. http://www.bioinformatics.babraham.ac.uk/proje cts/fastqc/ (2010).

45. Bolger, A. M., Lohse, M. \& Usadel, B. Trimmomatic: A flexible trimmer for Illumina sequence data. Bioinformatics 30, 2114-2120 (2014).

46. Kim, D., Langmead, B. \& Salzberg, S. L. HISAT: A fast spliced aligner with low memory requirements. Nat. Methods 12, 357-360 (2015).

47. Pertea, M. et al. StringTie enables improved reconstruction of a transcriptome from RNA-seq reads. Nat. Biotechnol. 33(3), 290-295 (2015).

48. Barbosa, S., Black, W. C. \& Hastings, I. Challenges in estimating insecticide selection pressures from mosquito field data. PLoS Negl. Trop. Dis. 5(11), e1387 (2011).

49. Reiner, R. C. Jr. et al. A systematic review of mathematical models of mosquito-borne pathogen transmission: 1970-2010. J. R. Soc. Interface 10(81), 20120921 (2013).

50. Baumann, P., Clark, M. A., Baumann, L. \& Broadwell, A. Bacillus sphaericus as a mosquitocidal pathogen: Properties of the organism and its toxins. Microbiol. Rev. 55, 425-436 (1991).

51. Charles, J. F., Nielsen-LeRoux, C. \& Delécluse, A. Bacillus sphaericus toxins: Molecular biology and mode of action. Ann. Rev. Entomol. 41, 451-472 (1996).

52. Li, S., De Mandal, S., Xu, X. \& Jin, F. The tripartite interaction of host immunity-Bacillus thuringiensis infection-gut microbiota. Toxins (Basel) 12(8), $514(2020)$.

53. Bierbaum, G. \& Sahl, H. G. Induction of autolysis of Staphylococci by the basic peptide antibiotic Pep5 and nisin and their influence on the activity of autolytic enzymes. Arch. Microbiol. 141, 249-254 (1985).

54. Bierbaum, G. \& Sahl, H. G. Autolytic system of Staphylococcus simulans 22: Influence of cationic peptides on activity of N-acetylmuramoyl-L-alanine amidase. J. Bacteriol. 169, 5452-5458 (1987).

55. Takatsuka, J. \& Kunimi, Y. Intestinal bacteria affect growth of Bacillus thuringiensis in larvae of the oriental tea tortrix, Homona magnanima diakonoff (Lepidoptera: Tortricidae). J. Invertebr. Pathol. 76(3), 222-226 (2000).

56. Shan, Y. et al. Cultivable gut bacteria of scarabs (Coleoptera: Scarabaeidae) inhibit Bacillus thuringiensis multiplication. Environ. Entomol. 43(3), 612-616 (2014).

57. Paramasiva, I., Sharma, H. C. \& Krishnayya, P. V. Antibiotics influence the toxicity of the delta endotoxins of Bacillus thuringiensis towards the cotton bollworm, Helicoverpa armigera. BMC Microbiol. 14, 200 (2014).

58. Visweshwar, R., Sharma, H. C., Akbar, S. M. \& Sreeramulu, K. Elimination of gut microbes with antibiotics confers resistance to Bacillus thuringiensis toxin proteins in Helicoverpa armigera (Hubner). Appl. Biochem. Biotech. 177(8), 1621-1637 (2015).

59. Regode, V., Kuruba, S., Mohammad, A. S. \& Sharma, H. C. Isolation and characterization of gut bacterial proteases involved in inducing pathogenicity of Bacillus thuringiensis toxin in cotton bollworm, Helicoverpa armigera. Front. Microbiol. 7, 1567 (2016).

60. Acharya, U. \& Acharya, J. K. Enzymes of sphingolipid metabolism in Drosophila melanogaster. Cell Mol. Life Sci. 62(2), 128-142 (2005).

61. Kerstholt, M. et al. Role of glutathione metabolism in host defense against Borrelia burgdorferi infection. Proc. Natl. Acad. Sci. USA 115(10), E2320-E2328 (2018).

62. Lemaitre, B. \& Hoffmann, J. The host defense of Drosophila melanogaster. Annu. Rev. Immunol. 25, 697-743 (2007).

63. Caccia, S. et al. Midgut microbiota and host immunocompetence underlie Bacillus thuringiensis killing mechanism. Proc. Natl. Acad. Sci. USA 113(34), 9486-9491 (2016).

\section{Acknowledgements}

This research was supported by National Natural Sciences Foundation of China (no. 81871686).

\section{Author contributions}

R.Z. and Z.Z. designed the study. W.L., Q.Z., and X.Z. carried out experiments and prepared mosquito samples. R.Z., W.L., Q.Z., and X.Z. analyzed data together. W.L, Q.Z., and, X.Z. prepared the original figures and tables. R.Z. and Z.Z. wrote the original draft of the paper. All authors contributed to reviewing and editing the paper.

\section{Competing interests}

The authors declare no competing interests.

\section{Additional information}

Supplementary Information The online version contains supplementary material available at https://doi.org/ 10.1038/s41598-021-99733-8.

Correspondence and requests for materials should be addressed to R.Z. or Z.Z.

Reprints and permissions information is available at www.nature.com/reprints.

Publisher's note Springer Nature remains neutral with regard to jurisdictional claims in published maps and institutional affiliations.

Open Access This article is licensed under a Creative Commons Attribution 4.0 International License, which permits use, sharing, adaptation, distribution and reproduction in any medium or format, as long as you give appropriate credit to the original author(s) and the source, provide a link to the Creative Commons licence, and indicate if changes were made. The images or other third party material in this article are included in the article's Creative Commons licence, unless indicated otherwise in a credit line to the material. If material is not included in the article's Creative Commons licence and your intended use is not permitted by statutory regulation or exceeds the permitted use, you will need to obtain permission directly from the copyright holder. To view a copy of this licence, visit http://creativecommons.org/licenses/by/4.0/.

(C) The Author(s) 2021 